\title{
Aquiescência em Autorrelatos de Personalidade: Uma Comparação de Métodos
}

\author{
Cristian Zanon ${ }^{1}$ \\ Universidade Federal do Rio Grande do Sul, Porto Alegre-RS, Brasil \\ João Paulo Araújo Lessa \\ Universidade São Francisco, Campus Swift, Campinas-SP, Brasil \\ Letícia Lovato Dellazzana-Zanon \\ Pontifícia Universidade Católica de Campinas, Campinas-SP, Brasil
}

\section{RESUMO}

O uso de instrumentos de autorrelato para a avaliação da personalidade é uma forma rápida, prática e econômica para esse fim. Contudo, o estilo de resposta aquiescente, que é a forma do indivíduo concordar com o item independentemente do conteúdo, pode impactar negativamente nas propriedades psicométricas desses instrumentos. O objetivo deste estudo foi investigar soluções fatoriais para avaliação da estrutura da personalidade por meio de diferentes métodos para o controle de estilo de resposta. $O$ primeiro método, mais simples e fácil de ser implementado, desenvolvido por Soto el al., (2008), e o segundo, mais complexo e sofisticado, foi desenvolvido por Maydeu-Olivares e Coffman (2006). Compuseram a amostra deste estudo 433 universitários que responderam à versão brasileira do Big Five Inventory. Os resultados demonstram que o controle de aquiescência pode ser essencial para adequada avaliação da personalidade e que o método de Maydeu-Olivares e Coffman apresentou solução fatorial mais teoricamente compatível com o Big Five. Implicações dos achados são apresentados neste estudo.

Palavras-chave: validade; Big Five; aquiescência.

\section{ABSTRACT - Acquiescence in self-report personality assessment: A comparison between methods}

The use of self-report instruments to assess personality is widely known for being efficient, practical and economical. However, acquiescent responding, which is the tendency of participants to endorse items regardless of their content, may negatively impact on the psychometric properties of these instruments. The objective of this study was to evaluate factor models of personality structure by using different methods for the control of response styles. The first method, simpler and easier to implement, was developed by Soto et al., (2008), and the second method, more complex and sophisticated, was developed by Maydeu-Olivares and Coffman (2006). The sample was composed by 433 undergraduate students who answered the Brazilian version of the Big Five Inventory. Results revealed that controlling for acquiesce response style is essential for a proper personality assessment, and that the Maydeu-Olivares and Coffman's method presented a more theoretically acceptable solution for the Big Five model. Implications of the findings are discussed.

Keywords: validity; Big Five; Acquiescence.

\section{RESUMEN - Aquiescencia en autoinforme de personalidad: Una comparación entre métodos}

El uso de instrumentos de autoinforme para evaluar la personalidad es ampliamente conocido por su eficacia, forma práctica y económica para este fin. No obstante, la aquiescencia, que es la tendencia de los participantes a respaldar ítems independientemente de su contenido, puede impactar negativamente en las propiedades psicométricas de estos instrumentos. La finalidad de este estudio fue investigar soluciones factoriales para la evaluación de la estructura de la personalidad a través de diferentes métodos para el control de estilo de respuesta. El primer método, más simple y más fácil de implementarse, fue desarrollado por Soto el al. (2008) y el segundo método, más complejo y sofisticado, fue desarrollado por Maydeu-Olivares y Coffman (2006). La muestra estuvo compuesta por 433 estudiantes universitarios que respondieron a la versión brasileña del Big Five Inventory. Los resultados demuestran que el control del estilo de la respuesta aquiescente es esencial para una evaluación de personalidad adecuada, y que el método de Maydeu-Olivares y Coffman presentó una solución teóricamente más compatible con el Big Five. En este estudio se discuten las implicaciones de los hallazgos.

Palabras clave: validez; Big Five; aquiescencia.

A avaliação de personalidade por meio de instrumentos de autorrelato compostos por escalas do tipo Likert constituem uma forma válida, rápida e com baixo custo para atingir esse objetivo. Contudo, a aquiescência, que é caracterizada pela concordância com os itens sem considerar o seu conteúdo, constitui um fenômeno 
conhecido há bastante tempo (Cronbach, 1946) que pode comprometer, consideravelmente, a adequada avaliação da personalidade pelo autorrelato (Rammstedt \& Farmer, 2013). Outros estilos de resposta, como discordar dos itens independentemente do seu conteúdo, evitar o uso dos pontos extremos, ou preferir os pontos extremos, também podem ameaçar a validade das avaliações (Paulhus, 1991).

Dentre os estilos de respostas mais estudados, a aquiescência é definida como a tendência do indivíduo em endossar itens que indicam quaisquer tipos de traços (Paulhus, 1991; Rammstedt \& Farmer, 2013). Por exemplo, um participante com um maior nível de aquiescência pode endossar itens que avaliam traços opostos, como ser feliz e ser triste. Ressalta-se que a avaliação de personalidade via autorrelato baseia-se no pressuposto de que o testando: (a) consegue compreender o conteúdo dos itens apresentados, (b) consegue refletir sobre o quanto aquelas características o descrevem (ou o quanto ele concorda com elas), e (c) consegue escolher no item um valor da escala tipo Likert que reflete o seu nível naquela determinada característica. Consequentemente, a não observância desses pressupostos podem resultar na produção de escores que não refletem a personalidade dos testandos, ou seja, escores inválidos (He, Bartram, Inceoglu, \& van de Vijver, 2014; Jackson \& Messick, 1958).

A aquiescência pode ser danosa às avaliações de personalidade porque pode comprometer as evidências de validade do construto investigado (e.g., produzir escores inapropriados) e limitar as inferências estatísticas sobre os parâmetros estimados por um determinado teste para uma população (Jackson \& Messick, 1958; Lechner \& Rammstedt, 2015). Em outras palavras, ela é capaz de produzir médias amostrais que não correspondem às populacionais. De forma geral, pode-se dizer que a aquiescência é uma ameaça à validade dos escores de inventários/escalas de personalidade por não necessariamente refletir o traço latente do participante. Evidências recentes indicam que o estilo de resposta aquiescente pode enviesar as cargas fatoriais dos itens e pode fazer com que a escala geral apresente parâmetros inapropriados, em especial, se ela for construída com itens de polo positivo (e.g.: "Eu me vejo como uma pessoa sociável") (Danner, Aichholzer, \& Rammstedt, 2015).

Alguns estudos indicam que a aquiescência apresenta uma relação negativa com a habilidade cognitiva dos participantes (He et al., 2014; Lechner \& Rammstedt, 2015; Rammstedt \& Farmer, 2013; Vigil-Colet, RuizPamies, Anguiano-Carrasco, \& Lorenzo-Seva, 2012). Ao estratificarem os participantes em grupos com diferentes níveis de habilidade cognitiva, os autores obtiveram resultados que suportam a hipótese de que a habilidade cognitiva constitui um dos fatores determinantes para a aquiescência. O processo adequado de resposta aos itens requer um alto nível de processamento cognitivo (e.g., atenção, compreensão, memória, tomada de decisão), sendo que o baixo nível cognitivo dos participantes pode produzir considerável variabilidade nas propriedades psicométricas dos instrumentos de autorrelato de personalidade, como validade e fidedignidade (Toomela, 2003; McLarnon \& Carswell, 2013). Na mesma linha de raciocínio, verificou-se que os baixos níveis de proficiência e socioeconômico de adultos estão associados a maiores níveis de aquiescência (Distefano, Morgan, \& Motl, 2012).

Outra variável associada à aquiescência é a idade. Um estudo de larga escala buscou avaliar a relação entre essas variáveis, identificando que os maiores níveis de aquiescência encontram-se em jovens entre 10 e 20 anos (Soto, John, Gosling, \& Potter, 2008), sendo maior nos mais novos. Com uma amostra total de mais de 230 mil jovens, os pesquisadores sugerem que esse resultado foi observado porque adolescentes mais velhos possuem um autoconceito mais claro de si, assim como maiores níveis de habilidades cognitivas (e.g., extensão de vocabulário e compreensão verbal) e por já estarem mais acostumados com modelos de questionários informais (e.g., encontrados em revistas e sites da internet) e formais (e.g., provas escolares). Uma vez que a aquiescência pode afetar substancialmente a validade e fidedignidade das avaliações de personalidade, alguns métodos para controlá-la foram propostos.

\section{Métodos para Controle dos Estilos de Resposta}

Dois procedimentos têm sido usados para controlar aquiescência. Um vale-se de procedimentos mais simples e facilmente implementados, enquanto o outro é implementado em uma abordagem de structural equation modeling (SEM). O presente estudo buscou investigar a adequação e possível vantagem desses procedimentos sobre o uso de escores brutos para avaliação da personalidade no contexto brasileiro.

Soto et al. (2008) propuseram uma forma de produzir escores "limpos" das respostas aquiescentes que requer o uso de itens positivos e negativos de personalidade para avaliação de cada fator. Para obtenção desses escores, é preciso: (a) selecionar pares de itens positivos e negativos (e.g., tímido x extrovertido, calmo x nervoso), (b) calcular uma média e desvio padrão geral para o grupo de pares de itens opostos em cada fator, (c) subtrair a média obtida de todas as respostas dadas para cada item, e (d) dividi-las pelo desvio padrão produzido anteriormente. Esse procedimento fará com que o conjunto de respostas tenha uma média igual a 0,0 e o desvio padrão igual a 1,0. Quanto mais alto o escore, mais elevado é o valor do traço latente de cada participante e vice-versa.

Em seu estudo, Soto et al. (2008) apresentam scree plots do Big Five apresentando os eigenvalues produzidos nas análises fatoriais realizadas por idade dos participantes para a solução bruta (não controlando a aquiescência) e a solução que utilizou o procedimento anteriormente descrito (controlando a aquiescência). 
Claramente, pode-se visualizar a existência de seis fatores na solução bruta, mas, quando se controla a aquiescência, o conjunto de cinco fatores aparece de forma distinta dos demais fatores. Os autores interpretam esse achado indicando que o sexto fator representa a aquiescência dos participantes.

Maydeu-Olivares e Coffman (2006) propuseram o modelo do fator comum com intercepto randômico que modela diferenças individuais sistemáticas não contempladas pelos fatores comuns. Os autores indicam que o modelo de fator comum (método tradicional usado em análises fatoriais exploratórias e confirmatórias) assume que todos os interceptos que compõem as equações de estimação dos escores dos participantes são constantes. Em outras palavras, o modelo do fator comum assume que os participantes usam os pontos da escala Likert adequadamente, ou seja, relacionando seu traço latente ao conteúdo do item. Uma vez que estilos de respostas constituem um fenômeno que podem influenciar os escores em avaliações de autorrelato de personalidade com escalas de resposta do tipo Likert, esse pressuposto pode ser demasiadamente rígido.

O modelo do fator comum com intercepto randômico permite que os interceptos variem entre os participantes, ainda que sejam os mesmos para todos os itens. Esse modelo não produz um escore que reflete a diferença individual de cada participante, mas estima uma variância na forma de variável aleatória. Apesar desse modelo ter sido desenvolvido para análises fatoriais confirmatórias (AFC), recentemente, Aicholzer (2014) adaptou-o para aplicações com análises fatoriais exploratórias (AFE) - método aqui utilizado. O que torna esse método diferente dos demais é que ele não parte do pressuposto que os itens somente carregarão no seu fator predeterminado (Aicholzer, 2014).

Alguns estudos já avaliaram a adequação do modelo do fator comum com intercepto randômico em escalas de personalidade. Kam, Zhou, Zhang e Ho (2012) e Greaves, Houkamau e Sibley (2017), por exemplo, verificaram a presença do estilo de resposta aquiescente como um fator extra e, após realizarem o controle do estilo de resposta, verificaram estruturas de personalidade mais adequadas. Outros estudos sugerem que esse modelo pode ser promissor em escalas que passaram por adaptação transcultural porque podem aumentar o enviesamento dos parâmetros estimados (Greaves et al., 2017), ou quando for uma escala que contenha solução fatorial mais complexa (e.g., multidimensional) (Aicholzer, 2014), como é o caso do Big Five Inventory usado neste estudo.

Assim, o objetivo deste estudo foi avaliar soluções fatoriais para avaliação da estrutura da personalidade por meio de diferentes métodos para o controle de estilo de resposta. Mais especificamente, investigou-se: (a) a pertinência da necessidade do controle de aquiescência em avaliações de personalidade via autorrelatos e (b) qual dos dois métodos descritos anteriormente produzem melhor estrutura fatorial de personalidade em termos de itens carregando nos fatores teoricamente esperados. Duas hipóteses guiam esta investigação:

- H1 - Espera-se que os métodos que controlam a aquiescência dos participantes produzam estruturas de personalidade mais adequadas teoricamente que os métodos que não controlam.

- H2 - Espera-se que o modelo do fator comum com intercepto randômico apresente estrutura de personalidade mais adequada que o método de Soto et al. (2008).

\section{Método}

\section{Participantes}

Uma amostra com 433 universitários foi coletada de forma grupal em uma universidade do interior do estado de São Paulo. A idade dessa amostra variou com participantes entre 17 e 36 anos, ambos os sexos. A média de idade dos participantes foi de 21 anos $(D P=2,5)$, sendo $68 \%$ do sexo feminino. Todos os participantes foram escolhidos por conveniência e sua participação foi voluntária.

\section{Instrumentos}

Para o presente estudo, o seguinte instrumento foi utilizado o Big Five Inventory - BFI - criado originalmente por John, Donahue e Kentle (1991) e composto por 44 itens, a serem respondidos em escala do tipo Likert de cinco pontos, sendo pontuado 1 para "discordo totalmente" e 5 para "concordo totalmente". Ele foi adaptado e validado para o Brasil por Andrade (2008). Os itens dessa escala são referentes aos Cinco Grandes Fatores: Abertura, Extroversão, Socialização, Neuroticismo e Conscienciosidade. Os coeficientes $\alpha$ de consistência interna dos fatores variam de 0,75 a 0,90 , com média 0,80 (para os Estados Unidos e Canadá) e os índices de teste-reteste variam de 0,80 a 0,90 , com média 0,85 (intervalo de três meses entre as aplicações, também para os Estados Unidos e Canadá) (John \& Srivasta, 1999).

\section{Procedimento}

Todas as escalas respondidas foram coletadas e agrupadas em um banco de dados. Em um primeiro momento, foi realizado um rapport de apresentação da pesquisa e dos procedimentos éticos, explicitando o caráter voluntário e não obrigatório, bem como a garantia de anonimato das informações, como consta na resolução 510 de 2016 do Conselho Nacional de Saúde (Brasil, 2016). Os estudantes que concordaram em fazer parte do estudo assinaram o Termo de Consentimento Livre e Esclarecido. Todos os procedimentos requeridos pelo Comitê de Ética da Universidade São Francisco foram atendidos pelo protocolo $\mathrm{n}^{\mathrm{o}}$ 39314114.4.0000.5514. 
E seguida, foi solicitado aos participantes que respondessem aos itens da escala de acordo com as instruções dadas e suas opiniões. A coleta de dados foi realizada em uma única sessão para cada turma de estudantes, com duração média de 25 minutos.

\section{Análise de Dados}

Foram realizadas quatro análises fatoriais utilizando diferentes procedimentos. A primeira utilizou o método de extração de fatores principal axis factoring com os escores brutos obtidos. Esse método foi selecionado por não assumir normalidade nos dados. $\mathrm{Na}$ segunda análise, o mesmo método de extração foi utilizado, porém foi realizada com os escores controlados por aquiescência por meio do método de Soto et al. (2008). Ambas as análises foram conduzidas no programa SPSS IBM e utilizaram a rotação Promax - método oblíquo que estima as intercorrelações entre os fatores. A terceira análise utilizou o método de extração MLR (maximum likelihood with robust standard errors) com os escores brutos obtidos. Por fim, a quarta análise avaliou o modelo do intercepto randômico com os escores brutos obtidos também utilizando o MLR. Essas duas últimas análises foram conduzidas no programa MPlus 7.1 e utilizaram a rotação Geomin que estima as intercorrelações entre os fatores. $\mathrm{O}$ único fator não correlacionado com os demais foi o do intercepto randômico no último modelo.

Buscou-se avaliar principalmente nessas quatro soluções a adequação das cargas fatoriais produzidas. $\mathrm{Ou}$ seja, em que medida os itens carregam no fator esperado ou não. Nas duas primeiras análises, os scree plots são apresentados para indicar visualmente a representação dos fatores extraídos. Por fim, avaliaram-se os índices de ajuste produzidos pelas duas últimas soluções investigadas.

Os índices avaliados foram qui-quadrado $\left(\chi^{2}\right)$, Root Mean Square Error Of Approximation (RMSEA) e o Comparative Fit Index (CFI). Valores que indicam modelos bem ajustados são: $\chi^{2}>0,05$; RMSEA $<0,06$ e CFI $>0,95$. Valores que indicam modelos com ajuste aceitáveis são: $\chi^{2}>0,05$; RMSEA $<0,08$ e CFI $>0,90$. Uma vez que o $\chi^{2}$ é sensível ao tamanho amostral, muitos modelos adequadamente ajustados com amostras grandes podem apresentar valores significativos (Kline, 2011). Por isso, recomenda-se interpretação de mais de um índice para avaliação dos modelos.

\section{Resultados}

\section{Análise fatorial com Principal Axis Factoring e escores brutos}

Essa solução ( $\mathrm{KMO}=0,82$ e teste de Bartlett significativo) produziu 32 itens com carga acima de 0,30 no fator esperado de cada um dos itens, cinco itens com carga dupla (no fator esperado e em outro não esperado), seis itens com cargas em fatores não esperados para estes itens, e um item não carregou em nenhum fator (Tabela 1). Quatro fatores apresentaram coeficientes alfa adequados (acima de 0,70), sendo que apenas o fator Socialização apresentou índice baixo $(\alpha<0,69)$ Figura 1 apresenta o scree plot dos eigenvalues dessa solução.

Tabela 1

Estrutura Fatorial do BFI Usando Principal Axis Factoring para Escores Brutos

\begin{tabular}{|c|c|c|c|c|c|}
\hline \multirow{2}{*}{ Itens } & \multicolumn{5}{|c|}{ Fatores } \\
\hline & A & $\mathrm{N}$ & $E$ & $\mathrm{C}$ & $\mathrm{S}$ \\
\hline bfi40_A & 0,71 & 0,06 & $-0,01$ & $-0,03$ & $-0,02$ \\
\hline bfi20_A & 0,66 & 0,05 & $-0,05$ & $-0,07$ & $-0,07$ \\
\hline bfi10_A & 0,65 & 0,04 & 0,01 & 0,05 & $-0,08$ \\
\hline bfi5_A & 0,61 & $-0,06$ & $-0,10$ & 0,05 & $-0,05$ \\
\hline bfi25_A & 0,58 & $-0,07$ & 0,04 & $-0,03$ & $-0,07$ \\
\hline bfi44_A & 0,53 & $-0,09$ & 0,06 & $-0,16$ & $-0,02$ \\
\hline bfi15_A & 0,51 & 0,21 & 0,16 & 0,06 & 0,08 \\
\hline bfi16_E & 0,48 & $-0,08$ & $-0,25$ & 0,08 & 0,16 \\
\hline bfi30_A & 0,44 & $-0,19$ & 0,09 & $-0,14$ & 0,05 \\
\hline bfi26_E & 0,41 & $-0,08$ & $-0,15$ & 0,08 & 0,04 \\
\hline bfi11_E & 0,37 & $-0,12$ & $-0,28$ & 0,16 & 0,04 \\
\hline bfi41_A & $-0,30$ & 0,20 & $-0,09$ & 0,12 & 0,11 \\
\hline bfi39_N & 0,02 & 0,81 & $-0,10$ & 0,04 & $-0,08$ \\
\hline bfi29_N & $-0,03$ & 0,68 & 0,11 & $-0,00$ & $-0,06$ \\
\hline bfi14_N & 0,04 & 0,63 & 0,15 & 0,09 & 0,21 \\
\hline bfi9_N & 0,24 & $-0,63$ & 0,21 & 0,07 & 0,07 \\
\hline bfi24_N & 0,24 & $-0,55$ & 0,12 & $-0,06$ & 0,14 \\
\hline bfi19_N & $-0,05$ & 0,45 & 0,15 & $-0,06$ & 0,39 \\
\hline bfi34_N & 0,26 & $-0,47$ & 0,12 & 0,05 & 0,13 \\
\hline
\end{tabular}


Tabela 1 (continuação)

Estrutura Fatorial do BFI Usando Principal Axis Factoring para Escores Brutos

\begin{tabular}{|c|c|c|c|c|c|}
\hline \multirow{2}{*}{ Itens } & \multicolumn{5}{|c|}{ Fatores } \\
\hline & A & $\mathrm{N}$ & $E$ & $\mathrm{C}$ & S \\
\hline bfi4_N & $-0,08$ & 0,40 & 0,26 & $-0,07$ & 0,05 \\
\hline bfi2_s & 0,17 & 0,37 & $-0,07$ & $-0,07$ & $-0,05$ \\
\hline bfi12_s & 0,24 & 0,35 & $-0,21$ & $-0,03$ & 0,04 \\
\hline bfi37_s & 0,24 & 0,33 & $-0,07$ & $-0,09$ & $-0,24$ \\
\hline bfi21_E & 0,05 & $-0,04$ & 0,83 & 0,04 & 0,03 \\
\hline bfi31_E & 0,06 & 0,08 & 0,80 & $-0,09$ & 0,06 \\
\hline bfi6_E & 0,14 & $-0,01$ & 0,72 & 0,04 & $-0,04$ \\
\hline bfi1_E & 0,22 & 0,14 & $-0,66$ & $-0,11$ & 0,05 \\
\hline bfi36_E & 0,20 & $-0,03$ & $-0,60$ & $-0,04$ & 0,16 \\
\hline bfi8_c & 0,31 & $-0,01$ & 0,05 & $-0,61$ & $-0,01$ \\
\hline bfi33_c & 0,14 & 0,05 & 0,11 & 0,54 & 0,08 \\
\hline bfi23_c & 0,08 & 0,20 & $-0,02$ & $-0,51$ & 0,08 \\
\hline bfi43_c & 0,11 & 0,17 & $-0,01$ & $-0,45$ & 0,18 \\
\hline bfi18_c & 0,29 & 0,03 & 0,06 & $-0,49$ & 0,07 \\
\hline bfi3_c & 0,14 & 0,23 & 0,06 & 0,46 & 0,09 \\
\hline bfi28_c & 0,23 & 0,02 & 0,05 & 0,46 & $-0,01$ \\
\hline bfi13_c & 0,38 & $-0,04$ & $-0,01$ & 0,39 & $-0,02$ \\
\hline bfi38_c & 0,32 & 0,05 & $-0,03$ & 0,39 & 0,04 \\
\hline bfi32_s & 0,03 & $-0,01$ & $-0,06$ & $-0,05$ & 0,63 \\
\hline bfi42_s & $-0,01$ & 0,06 & $-0,01$ & 0,10 & 0,59 \\
\hline bfi22_s & $-0,09$ & $-0,03$ & $-0,09$ & $-0,21$ & 0,57 \\
\hline bfi7_s & $-0,02$ & $-0,01$ & $-0,02$ & 0,02 & 0,53 \\
\hline bfi17_s & 0,02 & $-0,28$ & 0,08 & $-0,08$ & 0,46 \\
\hline bfi27_s & 0,44 & 0,08 & 0,18 & $-0,10$ & $-0,44$ \\
\hline bfi35_A & 0,07 & 0,06 & 0,15 & 0,09 & 0,21 \\
\hline Alfa & 0,79 & 0,80 & 0,85 & 0,73 & 0,62 \\
\hline
\end{tabular}

Nota. A=Abertura; E=Extroversão; N=Neuroticismo; C=Conscienciosidade; S=Socialização. Valores em negrito equivalem a cargas fatoriais maiores em módulo a 0,30. O alfa foi computado considerando apenas os itens teoricamente esperados para o fator.

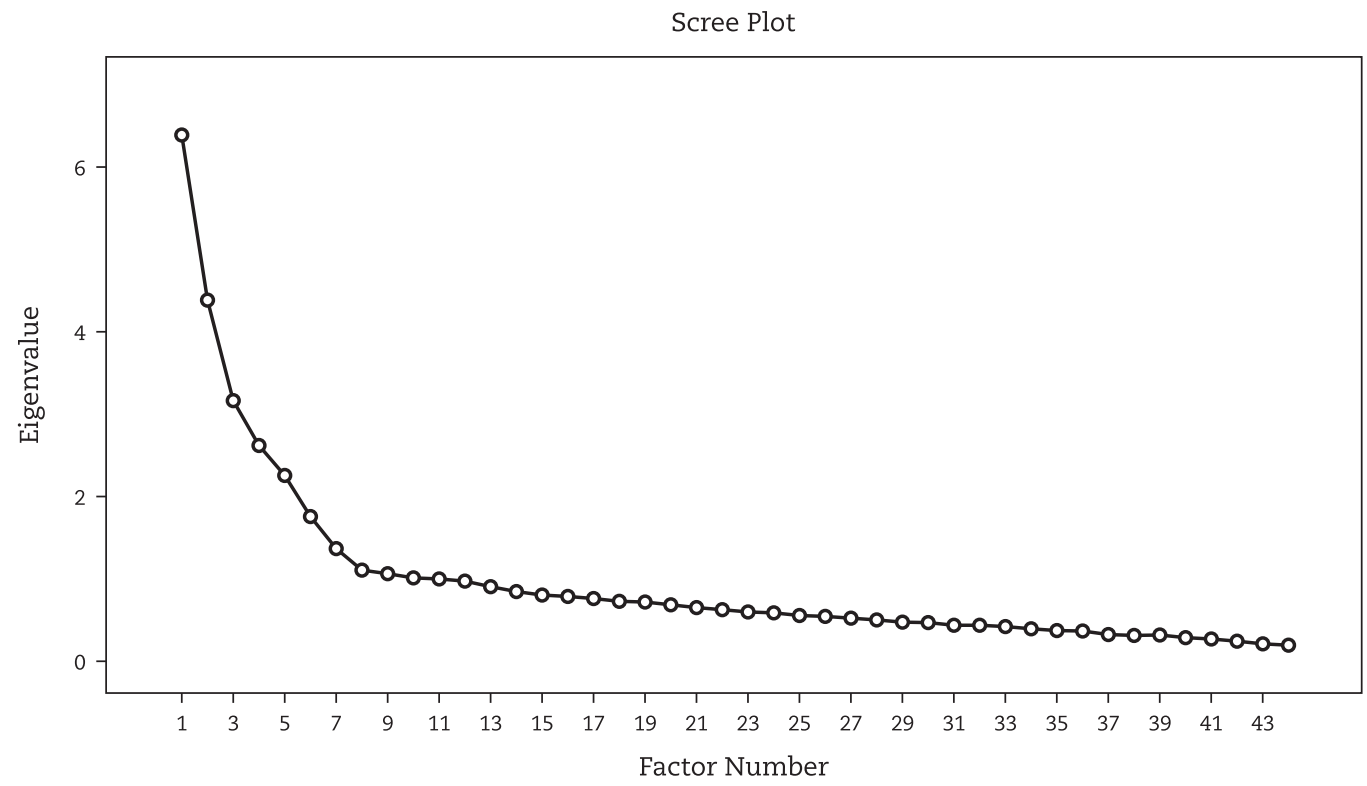

Figura 1. Scree plot dos eigenvalues produzidos pelo método principal axis factoring para escores brutos 


\section{Análise fatorial com principal axis factoring} e escores com aquiescência controlada

Essa solução produziu resultados superiores aos produzidos pelo método anterior, já que 36 itens com carga acima de 0,30 no fator esperado de cada um dos itens, um item com carga dupla (no fator esperado e em outro não esperado), cinco itens com cargas em fatores não esperados para os itens e dois itens não carregaram em nenhum fator (Tabela 2). Figura 2 apresenta o scree plot dos eigenvalues dessa solução.

Tabela 2

Estrutura Fatorial do BFI Usando Principal Axis Factoring para Escores Recodificados para Aquiescência

\begin{tabular}{|c|c|c|c|c|c|}
\hline \multirow{2}{*}{ Itens } & \multicolumn{5}{|c|}{ Fatores } \\
\hline & $\mathrm{A}$ & $E$ & $\mathrm{~N}$ & $\mathrm{C}$ & $\mathrm{S}$ \\
\hline bfi30_A & 0,80 & $-0,27$ & $-0,02$ & $-0,28$ & 0,19 \\
\hline bfi41_A & $-0,74$ & 0,38 & 0,12 & 0,39 & 0,10 \\
\hline bfi44_A & 0,74 & $-0,11$ & $-0,02$ & $-0,27$ & 0,02 \\
\hline bfi40_A & 0,73 & 0,06 & 0,03 & $-0,03$ & $-0,14$ \\
\hline bfi20_A & 0,70 & 0,10 & 0,06 & $-0,03$ & $-0,15$ \\
\hline bfi10_A & 0,66 & 0,05 & 0,03 & 0,09 & $-0,08$ \\
\hline bfi25_A & 0,59 & 0,04 & $-0,06$ & 0,07 & $-0,06$ \\
\hline bfi15_A & 0,58 & $-0,14$ & 0,21 & 0,09 & $-0,01$ \\
\hline bfi5_A & 0,53 & 0,16 & $-0,06$ & 0,14 & $-0,11$ \\
\hline bfi16_E & 0,45 & 0,23 & $-0,06$ & 0,06 & 0,17 \\
\hline bfi26_E & 0,32 & 0,16 & $-0,05$ & 0,21 & 0,11 \\
\hline bfi11_E & 0,31 & 0,29 & $-0,13$ & 0,23 & 0,04 \\
\hline bfi21_E & 0,01 & $-0,86$ & 0,03 & 0,33 & 0,02 \\
\hline bfi31_E & 0,09 & $-0,85$ & 0,14 & 0,13 & 0,08 \\
\hline bfi6_E & 0,11 & $-0,74$ & 0,04 & 0,30 & $-0,07$ \\
\hline bfi1_E & 0,15 & 0,69 & 0,11 & $-0,11$ & 0,13 \\
\hline bfi36_E & 0,13 & 0,62 & $-0,06$ & $-0,04$ & 0,18 \\
\hline bfi12_s & 0,04 & 0,43 & 0,23 & 0,03 & $-0,14$ \\
\hline bfi39_N & 0,02 & 0,09 & 0,77 & 0,10 & $-0,08$ \\
\hline bfi29_N & $-0,03$ & $-0,03$ & 0,67 & 0,12 & $-0,07$ \\
\hline bfi9_N & 0,01 & $-0,06$ & $-0,64$ & 0,27 & 0,14 \\
\hline bfi14_N & 0,10 & $-0,07$ & 0,59 & 0,12 & 0,17 \\
\hline bfi24_N & 0,02 & 0,03 & $-0,54$ & 0,18 & 0,16 \\
\hline bfi34_N & 0,08 & 0,03 & $-0,47$ & 0,20 & 0,09 \\
\hline bfi4_N & 0,04 & $-0,21$ & 0,46 & 0,04 & 0,14 \\
\hline bfi19_N & 0,01 & $-0,12$ & 0,45 & $-0,05$ & 0,36 \\
\hline bfi2_s & 0,01 & 0,24 & 0,31 & 0,02 & $-0,06$ \\
\hline bfi37_s & 0,06 & 0,25 & 0,27 & $-0,01$ & $-0,24$ \\
\hline bfi33_c & 0,09 & $-0,09$ & 0,05 & 0,63 & 0,07 \\
\hline bfi8_C & 0,17 & 0,20 & $-0,04$ & $-0,63$ & 0,01 \\
\hline bfi43_c & 0,11 & 0,20 & 0,18 & $-0,56$ & 0,18 \\
\hline bfi28_C & 0,17 & $-0,02$ & 0,02 & 0,55 & 0,03 \\
\hline bfi3_c & 0,12 & $-0,07$ & 0,22 & 0,54 & 0,07 \\
\hline bfi18_C & 0,11 & 0,23 & 0,01 & $-0,53$ & 0,03 \\
\hline bfi23_c & $-0,01$ & 0,25 & 0,20 & $-0,52$ & 0,16 \\
\hline bfi13_c & 0,21 & 0,15 & $-0,08$ & 0,51 & $-0,08$ \\
\hline bfi38_c & 0,21 & 0,10 & 0,03 & 0,43 & 0,07 \\
\hline bfi35_A & $-0,09$ & $-0,01$ & 0,05 & 0,26 & 0,17 \\
\hline bfi22_s & $-0,06$ & 0,13 & 0,02 & $-0,16$ & 0,66 \\
\hline bfi32_s & 0,03 & 0,07 & 0,01 & 0,01 & 0,64 \\
\hline bfi27_s & 0,22 & 0,09 & 0,04 & 0,12 & $-0,63$ \\
\hline bfi42_s & 0,07 & $-0,01$ & 0,07 & 0,13 & 0,62 \\
\hline
\end{tabular}


Tabela 2 (continuação)

Estrutura Fatorial do BFI Usando Principal Axis Factoring para Escores Recodificados para Aquiescência

\begin{tabular}{lccccc}
\hline & \multirow{2}{*}{ Itens } & \multicolumn{5}{c}{ Fatores } \\
\cline { 2 - 6 } & A & E & N & C & S \\
\hline bfi7_S & 0,07 & 0,01 & 0,05 & 0,07 & $\mathbf{0 , 5 7}$ \\
bfi17_S & 0,01 & $-0,04$ & $-0,24$ & 0,01 & $\mathbf{0 , 5 3}$ \\
\hline
\end{tabular}

Nota. A=Abertura; E=Extroversão; N=Neuroticismo; C=Conscienciosidade; S=Socialização. Valores em negrito equivalem a cargas fatoriais maiores em módulo a 0,30

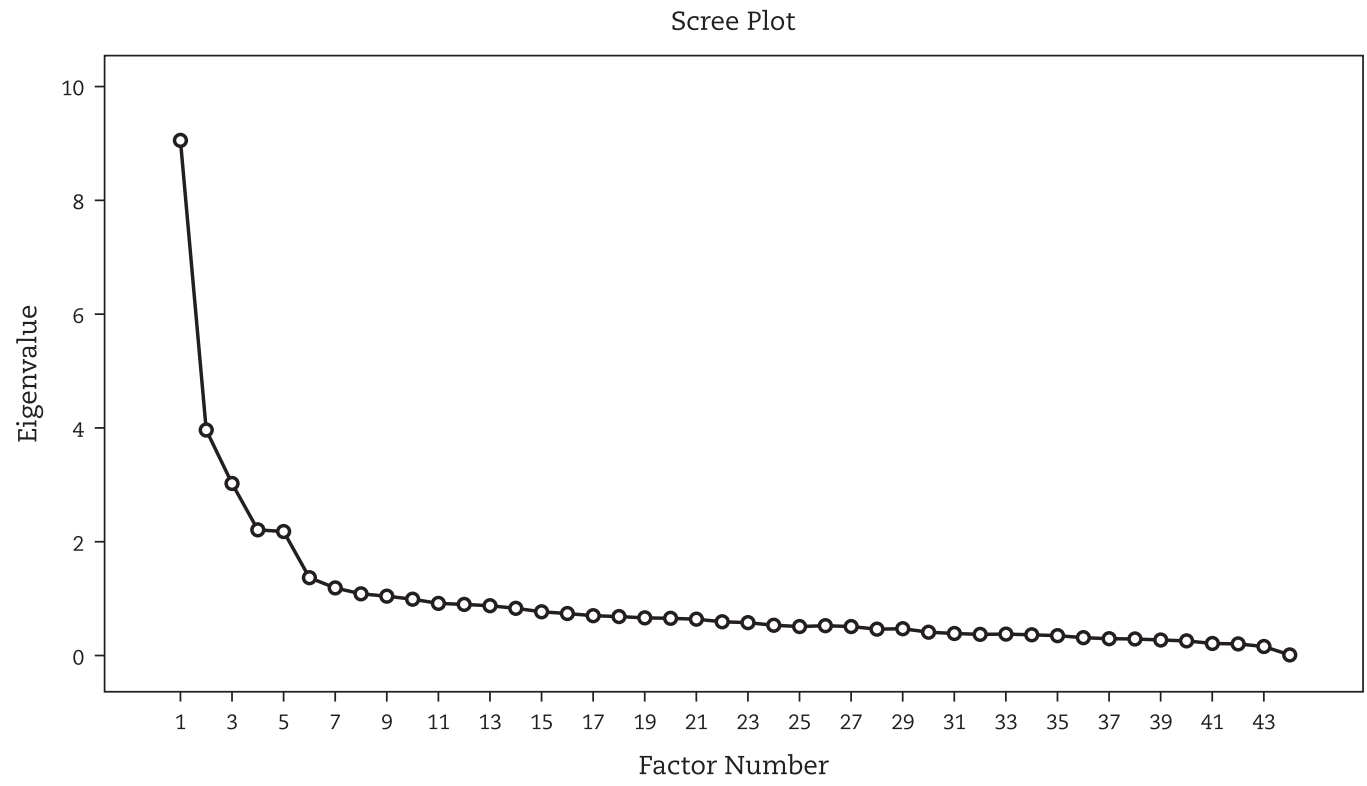

Figura 2. Scree plot dos eigenvalues produzidos pelo método principal axis factoring para escores controlados por aquiescência

\section{Análise fatorial com MLR e escores brutos}

Essa solução produziu a pior estrutura fatorial analisada neste estudo, já que identificou apenas 30 itens com carga acima de 0,30 no fator esperado para cada item, seis itens com carga dupla (no fator esperado e em outro não esperado), cinco itens com cargas em fatores não esperados para os itens e três itens não carregaram em nenhum fator (Tabela 3 ). Os índices de ajuste obtidos foram em parte insatisfatórios $\left(\chi^{2}<0,001\right.$ e CFI $=0,81)$ e em parte satisfatório (RMSEA $=0,047)$.

Tabela 3

Estrutura Fatorial do BFI Usando MLR para Escores Brutos

\begin{tabular}{lccccc}
\hline & \multirow{2}{*}{ Itens } & \multicolumn{3}{c}{ Fatores } \\
\cline { 2 - 6 } & A & E & N & C & S \\
\hline bfi10_A & $\mathbf{0 , 6 3}$ & $-0,05$ & $-0,01$ & 0,08 & $-0,08$ \\
bfi5_A & $\mathbf{0 , 6 3}$ & $-0,06$ & $-0,08$ & $-0,06$ & $-0,07$ \\
bfi20_A & $\mathbf{0 , 5 9}$ & 0,03 & $-0,08$ & 0,06 & $-0,05$ \\
bfi40_A & $\mathbf{0 , 5 8}$ & 0,01 & $-0,06$ & 0,05 & 0,12 \\
bfi16_E & $\mathbf{0 , 5 6}$ & $-0,03$ & $-0,24$ & $-0,05$ & 0,16 \\
bfi25_A & $\mathbf{0 , 5 5}$ & 0,07 & 0,05 & $-0,08$ & 0,02 \\
bfi15_A & $\mathbf{0 , 5 1}$ & $-0,02$ & 0,15 & 0,22 & 0,01 \\
bfi13_C & $\mathbf{0 , 5 0}$ & $-0,36$ & $-0,03$ & $-0,04$ & $-0,05$
\end{tabular}


Tabela 3 (continuação)

Estrutura Fatorial do BFI Usando MLR para Escores Brutos

\begin{tabular}{|c|c|c|c|c|c|}
\hline \multirow{2}{*}{ Itens } & \multicolumn{5}{|c|}{ Fatores } \\
\hline & A & $E$ & $\mathrm{~N}$ & $\mathrm{C}$ & $\mathrm{S}$ \\
\hline bfi11_E & 0,49 & $-0,13$ & $-0,28$ & 0,01 & 0,01 \\
\hline bfi38_c & 0,43 & $-0,30$ & $-0,06$ & 0,03 & 0,13 \\
\hline bfi26_E & 0,42 & $-0,08$ & $-0,15$ & $-0,04$ & 0,14 \\
\hline bfi44_A & 0,40 & 0,15 & 0,03 & $-0,05$ & $-0,01$ \\
\hline bfi8_c & 0,16 & 0,63 & 0,06 & $-0,04$ & $-0,07$ \\
\hline bfi18_C & 0,19 & 0,50 & 0,07 & 0,06 & 0,06 \\
\hline bfi23_c & $-0,01$ & 0,50 & $-0,02$ & 0,22 & 0,10 \\
\hline bfi43_c & 0,02 & 0,48 & $-0,03$ & 0,13 & 0,12 \\
\hline bfi28_c & 0,31 & $-0,39$ & 0,03 & $-0,01$ & 0,09 \\
\hline bfi33_c & 0,27 & $-0,41$ & 0,15 & 0,03 & 0,05 \\
\hline bfi3_c & 0,25 & $-0,42$ & 0,07 & 0,24 & 0,12 \\
\hline bfi21_E & $-0,01$ & 0,01 & 0,81 & $-0,05$ & 0,01 \\
\hline bfi31_E & 0,01 & 0,09 & 0,76 & 0,07 & $-0,04$ \\
\hline bfi6_E & 0,07 & 0,01 & 0,72 & $-0,01$ & $-0,02$ \\
\hline bfi36_E & 0,30 & 0,05 & $-0,56$ & 0,01 & 0,17 \\
\hline bfi1_E & 0,23 & 0,13 & $-0,63$ & 0,12 & 0,01 \\
\hline bfi39_N & 0,04 & $-0,03$ & 0,02 & 0,78 & $-0,16$ \\
\hline bfi29_N & 0,01 & 0,02 & 0,12 & 0,69 & $-0,06$ \\
\hline bfi14_N & 0,10 & $-0,04$ & 0,16 & 0,63 & 0,12 \\
\hline bfi19_N & $-0,04$ & 0,11 & 0,17 & 0,44 & 0,23 \\
\hline bfi4_N & $-0,06$ & 0,11 & 0,24 & 0,38 & 0,07 \\
\hline bfi24_N & 0,24 & 0,11 & 0,12 & $-0,55$ & 0,06 \\
\hline bfi34_N & 0,30 & $-0,03$ & 0,09 & $-0,34$ & 0,04 \\
\hline bfi9_N & 0,26 & 0,01 & 0,22 & $-0,58$ & $-0,02$ \\
\hline bfi42_s & 0,13 & 0,01 & $-0,01$ & 0,07 & 0,63 \\
\hline bfi37_s & 0,23 & 0,06 & $-0,04$ & 0,33 & $-0,41$ \\
\hline bfi27_s & 0,36 & 0,02 & 0,20 & 0,03 & $-0,58$ \\
\hline bfi7_s & 0,084 & $-0,02$ & $-0,03$ & 0,02 & 0,52 \\
\hline bfi32_s & 0,15 & 0,15 & $-0,03$ & 0,01 & 0,50 \\
\hline bfi22_s & $-0,01$ & 0,23 & $-0,06$ & $-0,02$ & 0,48 \\
\hline bfi17_s & 0,08 & 0,06 & 0,08 & $-0,25$ & 0,32 \\
\hline bfi30_A & 0,29 & 0,09 & 0,07 & $-0,02$ & 0,09 \\
\hline bfi35_A & 0,11 & $-0,04$ & 0,08 & 0,03 & 0,08 \\
\hline bfi2_s & 0,14 & 0,09 & $-0,11$ & 0,36 & $-0,02$ \\
\hline bfi41_A & $-0,12$ & $-0,07$ & $-0,01$ & 0,15 & $-0,09$ \\
\hline bfi12_s & 0,31 & 0,07 & $-0,16$ & 0,30 & $-0,09$ \\
\hline
\end{tabular}

Nota. A=Abertura; E=Extroversão; N=Neuroticismo; C=Conscienciosidade; S=Socialização. Valores em negrito equivalem a cargas fatoriais maiores em módulo a 0,30

\section{Análise fatorial com o modelo do intercepto randômico e escores brutos}

Essa solução produziu a melhor estrutura fatorial analisada neste estudo, já que identificou 37 itens com carga acima de 0,30 no fator esperado para cada item, dois itens com carga dupla (no fator esperado e em outro não esperado), dois itens com cargas em fatores não esperados para os itens e três itens não carregaram em nenhum fator (Tabela 4). Os índices de ajuste obtidos também foram em parte insatisfatórios $\left(\chi^{2}<0,001, \mathrm{CFI}=0,84\right)$ e em parte satisfatório $(\mathrm{RMSEA}=0,044)$. 
Tabela 4

Estrutura Fatorial do BFI Usando Modelo do Intercepto Randômico para Escores Brutos

\begin{tabular}{|c|c|c|c|c|c|}
\hline \multirow{2}{*}{ Itens } & \multicolumn{5}{|c|}{ Fatores } \\
\hline & $E$ & $\mathrm{~N}$ & $\mathrm{~A}$ & $\mathrm{C}$ & $S$ \\
\hline bfi1_E & 0,72 & 0,06 & 0,06 & $-0,10$ & 0,06 \\
\hline bfi21_E & $-0,78$ & $-0,07$ & $-0,01$ & 0,03 & 0,04 \\
\hline bfi36_E & 0,60 & $-0,02$ & 0,17 & 0,01 & $-0,11$ \\
\hline bfi31_E & $-0,71$ & 0,04 & $-0,01$ & $-0,07$ & 0,09 \\
\hline bfi6_E & $-0,71$ & $-0,01$ & 0,11 & 0,02 & 0,06 \\
\hline bfi11_E & 0,38 & $-0,16$ & 0,20 & 0,18 & 0,07 \\
\hline bfi16_E & 0,31 & $-0,08$ & 0,40 & 0,07 & $-0,06$ \\
\hline bfi39_N & 0,06 & 0,72 & $-0,07$ & 0,05 & 0,24 \\
\hline bfi29_N & $-0,05$ & 0,64 & $-0,08$ & $-0,02$ & 0,14 \\
\hline bfi14_N & $-0,08$ & 0,58 & 0,04 & 0,08 & $-0,01$ \\
\hline bfi34_N & 0,05 & $-0,43$ & $-0,02$ & 0,10 & 0,01 \\
\hline bfi24_N & $-0,01$ & $-0,63$ & $-0,02$ & $-0,05$ & $-0,02$ \\
\hline bfi9_N & $-0,10$ & $-0,66$ & $-0,05$ & 0,06 & 0,05 \\
\hline bfi19_N & $-0,10$ & 0,40 & $-0,04$ & $-0,07$ & $-0,14$ \\
\hline bfi4_N & $-0,23$ & 0,35 & 0,02 & $-0,11$ & $-0,03$ \\
\hline bfi40_A & 0,05 & 0,05 & 0,60 & $-0,01$ & $-0,04$ \\
\hline bfi20_A & 0,09 & 0,06 & 0,55 & $-0,04$ & 0,15 \\
\hline bfi44_A & $-0,04$ & 0,02 & 0,54 & $-0,16$ & $-0,16$ \\
\hline bfi5_A & 0,10 & $-0,07$ & 0,52 & 0,08 & 0,16 \\
\hline bfi10_A & 0,07 & 0,04 & 0,48 & 0,06 & 0,20 \\
\hline bfi30_A & $-0,15$ & 0,02 & 0,47 & $-0,13$ & $-0,06$ \\
\hline bfi25_A & $-0,02$ & $-0,09$ & 0,47 & $-0,05$ & 0,07 \\
\hline bfi15_A & $-0,08$ & 0,19 & 0,40 & 0,05 & 0,12 \\
\hline bfi41_A & 0,19 & 0,06 & $-0,47$ & 0,16 & 0,10 \\
\hline bfi26_E & 0,14 & $-0,03$ & 0,39 & 0,09 & $-0,08$ \\
\hline bfi8_c & 0,01 & $-0,10$ & 0,08 & $-0,57$ & 0,16 \\
\hline bfi33_c & $-0,05$ & $-0,01$ & 0,03 & 0,48 & 0,01 \\
\hline bfi3_c & $-0,02$ & 0,22 & 0,15 & 0,43 & $-0,07$ \\
\hline bfi13_c & 0,13 & $-0,09$ & 0,20 & 0,43 & 0,12 \\
\hline bfi28_c & 0,01 & $-0,02$ & 0,19 & 0,41 & $-0,06$ \\
\hline bfi18_c & $-0,01$ & $-0,01$ & 0,14 & $-0,47$ & 0,05 \\
\hline bfi23_c & 0,07 & 0,17 & 0,01 & $-0,50$ & $-0,03$ \\
\hline bfi43_c & 0,08 & 0,18 & 0,04 & $-0,43$ & $-0,11$ \\
\hline bfi38_c & 0,10 & 0,02 & 0,30 & 0,33 & $-0,06$ \\
\hline bfi27_s & $-0,10$ & $-0,05$ & 0,05 & $-0,08$ & 0,62 \\
\hline bfi37_s & 0,12 & 0,22 & $-0,12$ & $-0,01$ & 0,51 \\
\hline bfi32_s & 0,18 & $-0,07$ & 0,01 & $-0,06$ & $-0,41$ \\
\hline bfi22_s & 0,17 & $-0,07$ & $-0,09$ & $-0,15$ & $-0,41$ \\
\hline bfi7_s & 0,07 & 0,01 & 0,11 & 0,05 & $-0,44$ \\
\hline bfi42_s & 0,06 & 0,06 & 0,15 & 0,06 & $-0,55$ \\
\hline bfi2_s & 0,20 & 0,29 & 0,01 & $-0,07$ & 0,10 \\
\hline bfi17_s & 0,01 & $-0,29$ & $-0,03$ & 0,01 & $-0,26$ \\
\hline bfi35_A & 0,02 & $-0,03$ & $-0,08$ & 0,08 & $-0,03$ \\
\hline bfi12_s & 0,30 & 0,22 & 0,03 & 0,01 & 0,21 \\
\hline
\end{tabular}

Nota. A=Abertura; E=Extroversão; N=Neuroticismo; C=Conscienciosidade; S=Socialização. Valores em negrito equivalem a cargas fatoriais maiores em módulo a 0,30 


\section{Discussão}

Este estudo objetivou avaliar as possíveis vantagens do controle de estilos de resposta na avaliação de autorrelato de personalidade e também a pertinência das estruturas produzidas por dois métodos desenvolvidos para esse fim. De forma geral, os resultados indicam que as soluções que controlam estilos de resposta apresentam melhor estrutura fatorial, sendo que a solução produzida pelo modelo do fator comum com intercepto randômico parece mais adequada empírica e teoricamente.

Os resultados deste estudo estão de acordo com achados anteriores que indicam o controle da aquiescência como um método relevante para aumento das evidências de validade de autorrelato de personalidade (Soto et al., 2008). Este estudo evidencia que o uso do método proposto por Soto et al. (2008) em uma análise de eixos principais, produz estrutura fatorial mais robusta que a solução que não o utiliza. Na comparação dos scree plots (ver Figura 1 e Figura 2) produzidos em ambas as análises, pode-se visualizar, claramente, os cinco fatores presentes na análise que controla aquiescência (Figura 2). Ademais, o número de itens carregando nos fatores esperados foi ligeiramente superior e o número de itens carregando em fatores não esperados foi menor.

O modelo de Maydeu-Olivares e Coffman (2006), entretanto, parece ser o mais apropriado para controle de estilos de resposta. Os resultados produzidos com a utilização desse modelo foram superiores aos demais em termos do número de itens carregando no fator esperado e não carregando em fatores não esperados (que foi menor). Verificaram-se 37 itens carregando no fator esperado e apenas cinco itens foram inapropriados (em fator inesperado ou que não carregaram em nenhum fator). Uma explicação para a superioridade do método do intercepto randômico sobre os demais é que ele foi desenvolvido para controlar estilos de respostas variadas a escalas de tipo Likert (e.g., aquiescência, desaquiencência, preferência por pontos extremos, preferência por pontos médios, evitação de pontos extremos, entre outras), enquanto o método de Soto et al. (2008) foi desenvolvido para controlar aquiescência apenas.

Apesar da aquiescência ser o estilo de resposta mais estudado, desconhece-se se sua frequência é superior aos demais estilos apresentados. Sendo assim, a possibilidade de controlar estilos para além do aquiescente parece contribuir para estimação de estruturas fatoriais de personalidade. Esses resultados indicam a necessidade de revisões dos itens que carregaram em fatores inapropriados e que não carregaram em nenhum fator e nova reavaliação da estrutura fatorial do BFI no contexto brasileiro.

Os resultados deste estudo produzem evidências alinhadas com estudos prévios que indicam que controle de estilo de respostas constitui um procedimento importante no que diz respeito à validade e à fidedignidade dos escores de testes de personalidade (Aicholzer, 2014; Greaves et al., 2017; Kam et al., 2012). Por isso, sugere-se que esse método de controle para respostas aquiescentes seja utilizado tanto em pesquisas quanto em contextos aplicados (e.g., seleção e diagnóstico). O controle do estilo de resposta, mais amplo que a aquiescência, possibilitado pelo modelo do intercepto randômico permite a estimação de parâmetros mais acurados e, consequentemente, escores mais válidos, ou seja, os valores produzidos são mais prováveis de revelarem uma relação adequada com os traços latentes dos participantes.

Uma vez que subgrupos com características específicas, comumente, integram bancos de dados de pesquisa sobre personalidade (e.g., adolescentes, baixo nível cognitivo e baixo nível socioeconômico), é possível que alguns grupos apresentem escores mais distorcidos que outros em razão do nível de aquiescência presente nos participantes. O controle dos estilos de resposta pode minimizar estas distorções e produzir comparações mais justas e adequadas, quando for o caso.

Este estudo não está isento de limitações. Devido ao uso de uma amostra por conveniência composta por universitários, os resultados obtidos ficam impossibilitados de serem generalizados para a população geral. A não inclusão de grupos com níveis mais elevados de aquiescência como adolescentes de menor idade e pessoas com baixo nível cognitivo e menores níveis socioeconômico (Danner et al., 2015; Henrich, Heine, \& Norenzayan, 2010; McLarnon \& Carswell, 2013; Talhelm et al. 2015) não permite avaliar a adequação desses modelos para essas populações. Assim, sugere-se que estudos futuros envolvendo a avaliação da personalidade - por meios de escalas de tipo Likert - incluam grupos (a) com diferentes níveis de aquiescência e (b) mais fidedignos às configurações socioeconômicas do Brasil para avaliar a adequação dos métodos aqui apresentados.

\section{Referências}

Aicholzer, J. (2014). Random intercept EFA of personality scales. Journal of Research in Personality, 53, 1-4. doi: 10.1016/j.jrp.2014.07.001 Andrade, J. M. (2008). Evidências de validade do inventário dos cinco grandes fatores de personalidade para o Brasil (Tese de doutorado). Universidade de Brasília, Brasília, Brasil. Recuperado de: http://repositorio.unb.br/handle/10482/1751

Brasil, Conselho Nacional de Saúde. (2016). Resolução CNS 510, de 07 de abril de 2016. Recuperado de http://conselho.saude.gov.br/ resolucoes/2016/Reso510.pdf 
Cronbach,J.L.(1946). Response sets and testvalidity.EducationalandPsychologicalMeasurement, 6(4), 475-494.doi: 10.1177/001316444600600405

Danner, D., Aichholzer, J., \& Rammstedt, B. (2015). Acquiescence in personality questionnaires: Relevance, domain specificity, and stability. Journal of Research in Personality, 57, 119-130. doi: 10.1016/j.jrp.2015.05.004

DiStefano, C., Morgan, G. B., \& Motl, R. W. (2012). An examination of personality characteristics related to acquiescence. Journal of Applied Measurement, 13(1), 41-56. Recuperado de: https://www.researchgate.net/publication/225282558

Greaves, L. M., Houkamau, C. A., \& Sibley, C. G. (2017). Random intercept exploratory factor analysis of the multidemensional model of Maori identity and cultural engagement. Personality and Individual Differences, 105, 14-18. doi: 10.1016/j.paid.2016.09.026

He, J., Bartram, D., Inceoglu, I., van de Vijver, J. R. (2014). Response styles and personality traits: A mulitlevel analysis. Journal of Cross-Cultural Psychology, 45(7), 1028-1045. doi: 10.1177/0022022114534773

Henrich, J., Heine, S. J., \& Norenzayan, A. (2010). The weirdest people in the world. Behavioral and Brain Sciences, 33, 61-135. doi: 10.1017/ S0140525X0999152X

Jackson, D. N., \& Messick, S. (1958). Content and style in personality assessment. Psychological Bulletin, 55(4), 243-252. doi: 10.1037/ h0045996

John, O. P., Donahue, E. M., \& Kentle, R. L. (1991). The Big Five Inventory-Versions 4a and 54. Berkeley, CA: University of California, Berkeley, Institute of Personality and Social Research.

John, O. P., \& Srivasta, S. (1999). The Big-Five trait taxonomy: History, measurement, and theoretical perspectives. Em O. P. John \& L. A. Pervin, Handbook of Personality: Theory and Research (2a ed., pp. 102-138). New York: Guilfors Press.

Kam, C., Zhou, X., Zhang, X., \& Ho, M. Y. (2012). Examining the dimensionality of self-construals and individualistic-collectivistic values with random intercept item factor analysis. Personality and Individual Differences, 53(6), 727-733. doi: 10.1016/j.paid.2012.05.023

Kline, R. B. (2011). Convergence of structural equation modeling and multilevel modeling. Em M. Williams \& W. P. Vogt (Eds.), Handbook of methodological innovation in social research methods (pp. 562-589). London: Sage.

Lechner, C. M., \& Rammstedt, B. (2015). Cognitive ability, acquiescence, and the structure of personality in a sample of older adults. Psychological Assessment, 27, 1301-1311. doi: 10.1037/pas0000151

Maydeu-Olivares, A., \& Coffman, D. L. (2006). Random intercept item factor analsyis. Psychological Methods. 11, 344-362. doi: 10.1037/1082989X.11.4.344

McLarnon, M. J. W., \& Carswell, J. J. (2013). The personality differentiation by inteligence hypothesis: A measurement invariance investigation. Personality and Individual Differences, 54, 557-561. doi: 10.1016/j.paid.2012.10.029

Paulhus, D. L. (1991). Measurements and control of response bias. Em J. P. Robinson, P. R., Shaver \& L. S. Wrightsman (Eds.) Measures of Personality and Social Psychological Attitudes (pp. 17-59). San Diego: Academic Press. doi: 10.1016/B978-0-12-590241-0.50006-X

Rammstedt, B., \& Farmer, R. F. (2013). The impact of acquiescence on the evaluation of personality structure. Psychological Assessment, 25, 1137-1145. doi: 10.1037/a0033323

Soto, C. J., John, O. P., Gosling, S. D., \& Potter, J. (2008). The developmental psychometrics of big five self-reports: Acquiescence, factor structure, coherence, and differentiation from ages 10 to 20. Journal of Personality and Social Psychology, 94, 718-737. doi: 10.1037/00223514.94.4.718

Talhelm, T., Haidt, J., Oishi, S., Zhang, X., Miao, F. F., \& Chen, S. (2015). Liberals think more analytically (more "WEIRD”) than conservatives. Personality and Social Psychology Bulletin, 41, 250-267. doi: 10.1177/0146167214563672

Toomela, A. (2003). Relationships between personality structure, structure of word meaning, and cognitive ability: A study of cultural mechanisms of personality. Journal of Personality and Social Psychology, 85, 723-735. doi: 10.1037/0022-3514.85.4.723

Vigil-Colet, A., Ruiz-Pamies, M., Anguiano-Carrasco, C., \& Lorenzo-Seva, U. (2012). The impact of social desirability on psychometric measures of aggression. Psicothema, 24, 310-315. doi: 10.7334/psicothema2012.297

\section{Sobre os autores}

Cristian Zanon é psicólogo (UFSM), mestre, doutor e pós-doutor em Psicologia pela Universidade Federal do Rio Grande Sul. Atualmente é professor do Programa de Pós-Graduação em Psicologia da Universidade Federal do Rio Grande Sul.

João Paulo Araújo Lessa é psicólogo (CESMAC), mestre e doutorando em Psicologia pela Universidade São Francisco. Atualmente é consultor acadêmico do Instituto Ayrton Senna.

Letícia Lovato Dellazzana-Zanon é Doutora em Psicologia pela Universidade Federal do Rio Grande do Sul (UFRGS). Atualmente é Professora do Programa de Pós-Graduação em Psicologia da Pontifícia Universidade de Campinas (PUC-Campinas). 